\title{
Seeing The Solar Corona in Three Dimensions
}

\author{
Alberto M. Vásquez ${ }^{\mathrm{a}, \mathrm{b}, *}$ \\ ${ }^{a}$ Instituto de Astronomía y Física del Espacio (IAFE, CONICET-UBA), CC 67 - Suc \\ 28, (C1428ZAA) Ciudad de Buenos Aires, Argentina. \\ ${ }^{b}$ Facultad de Ciencias Exactas y Naturales (FCEN), University of Buenos Aires (UBA), \\ Ciudad de Buenos Aires, Argentina.
}

\begin{abstract}
The large availability and rich spectral coverage of today's observational data of the solar corona, and the high spatial and temporal resolution provided by many instruments, has enabled the evolution of three-dimensional (3D) physical models to a great level of detail. However, the 3D information provided by the data is rather limited as every instrument observes from a single angle of vision, or two at the most in the case of the STEREO mission. Two powerful available observational techniques to infer detailed 3D information of the solar corona from empirical data are stereoscopy and tomography. In particular, the technique known as differential emission measure tomography (DEMT) allows determination of the 3D distribution of the coronal electron density and temperature in the inner corona. This paper summarizes the main technical aspects of DEMT, reviews all published work based on it, and comments on its future development and applications.
\end{abstract}

Keywords: Corona, EUV, Tomography

\section{Introduction}

The solar corona can be observed in white light, EUV, X-ray, and radio wavelengths. Being the corona optically thin in these spectral ranges, its images are two-dimensional (2D) projections of the 3D emitting structure. Detailed knowledge of the 3D distribution of the fundamental plasma parameters of the solar corona $\left(\mathbf{B}, N_{e}, T_{e}\right)$ is highly desirable to advance

\footnotetext{
* Corresponding author

Email address: albert@iafe.uba.ar (Alberto M. Vásquez)
} 
its modeling. Stereoscopy and tomography are powerful observational techniques of the corona, that allow to infer quantitative $3 \mathrm{D}$ information. An excellent introduction to solar stereoscopy can be found in Inhester (2006). A recent general review on both techniques covering all the spectral ranges listed above can be found in Aschwanden (2011), with a strong focus on stereoscopy. In this review we specifically focus on differential emission measure tomography (DEMT) in a more extensive fashion, updating on all published work in the field at the moment of writing this article.

Minnaert (1930) originally developed the scattering theory of the photospheric white light (WL) by the free electrons of the corona, that allows to infer the 3D distribution of the coronal electron density from WL images. van de Hulst (1950) was the first to perform a global corona reconstruction using eclipse images and assuming full azimuthal axi-symmetry, an assumption firstly relaxed by Leblanc et al. (1970). It was Altschuler and Perry (1972) who developed the first actual solar rotational tomography (SRT) using coronagraph data. A good review on WL SRT can be found in Frazin (2000) and Frazin and Janzen (2002), who developed a robust, regularized, positive method for tomographic inversion of the coronal density from time series of WL images. Later on, Frazin et al. (2005) first introduced the concept of DEMT, a technique which uses time series of EUV images to determine the 3D distribution of the coronal local-DEM (or LDEM).

DEMT was developed by Frazin et al. (2009), and firstly applied by Vásquez et al. (2009) to study the 3D structure of coronal prominence cavities. The technique consists of two steps. In a first step the tomographic inversion of time series of full-sun EUV images is performed, to find the 3D distribution of the EUV emissivity in each filter band of the telescope. In a second step the emissivities found for all bands in any given coronal location are used as a constraint to infer the LDEM. Finally, moments of the LDEM are taken, as a result of which global 3D maps of the coronal electron density and temperature are produced.

In this review we summarize the main aspects and applications of the DEMT technique. Sections 2 and 3 describe and illustrate the two steps of DEMT, section 4 is a review of published results using the technique, and section 5 summarizes its main characteristics and future prospects for its development and application. 


\section{The Tomographic Model of the Corona}

To perform the EUV tomography, the inner corona volume in the height range $1.00-1.25 R_{\odot}$ is discretized on a $25 \times 90 \times 180$ (radial $\times$ latitudinal $\times$ longitudinal) spherical grid. Due to optical depth issues (analyzed in detail in Frazin et al. (2009)) and EUV signal-to noise levels (which depend on the particular filter), the results are reliable typically in the height range from 1.03 to $1.20 R_{\odot}$.

For each filter band of the EUV telescope separately, time series of fullsun EUV coronal images covering a complete solar rotation are used to find the $3 \mathrm{D}$ distribution of an emissivity-type quantity known as the filter band emissivity (FBE). The FBE of each EUV filter is the integral over wavelength of the coronal spectral emissivity multiplied by the passband of the filter. The intensity in each pixel is a line-of-sight integral of the FBE. The intensities of all pixels of all images can be arranged in a single very large column vector, as well as the FBE in every cell (or voxel) of the tomographic grid. In this way, both vectors are linearly related through a very large non-square sparse projection matrix, that depends on the geometry of the observations. Both the projection matrix and the pixel intensity vector are known, and the problem is to find the FBE vector. This poses a non-invertible linear problem for each band separately, which is the tomographic problem.

In the case both instruments the Extreme ultraviolet Imaging Telescope (EIT) on board the Solar and Heliospheric Observatory (SoHO), and the Extreme UltraViolet Imager (EUVI) on board the Solar TErrestrial RElations Observatory (STEREO), the number of EUV bands that can be used for DEMT is 3. In the case of the Atmospheric Imaging Assembly (AIA) on board the Solar Dynamics Observatory (SDO) the number of bands is increased to 6 .

The 3D distribution of the FBE is found by solving a global optimization problem, and the FBE distribution that best reproduces the intensities observed in all the pixels of all the images is determined. A thorough technical explanation of all aspects of the inversion can be found in Frazin et al. (2009), and discussions on the uncertainties involved can be found in Vásquez et al. (2009, 2010, 2011); Nuevo et al. (2015).

Based on reconstructions using data of the EUVI instrument, Figure 1 shows a summary of the EUV tomography step. The first column shows (from top to bottom) images in the bands of 171, 195 and $284 \AA$. These images are just one sample from the time series actually used to perform the 
tomography. For each band, the second through fourth columns show, in a color scale, projected spherical cuts of the tomographic FBE at 1.035, 1.085, and $1.135 R_{\odot}$, respectively. The last column shows the respective synthetic images calculated by integrating the tomographic models along the line-ofsight. The black streaks seen in the reconstructions near some of the active regions are artifacts caused by the Sun's temporal variability.

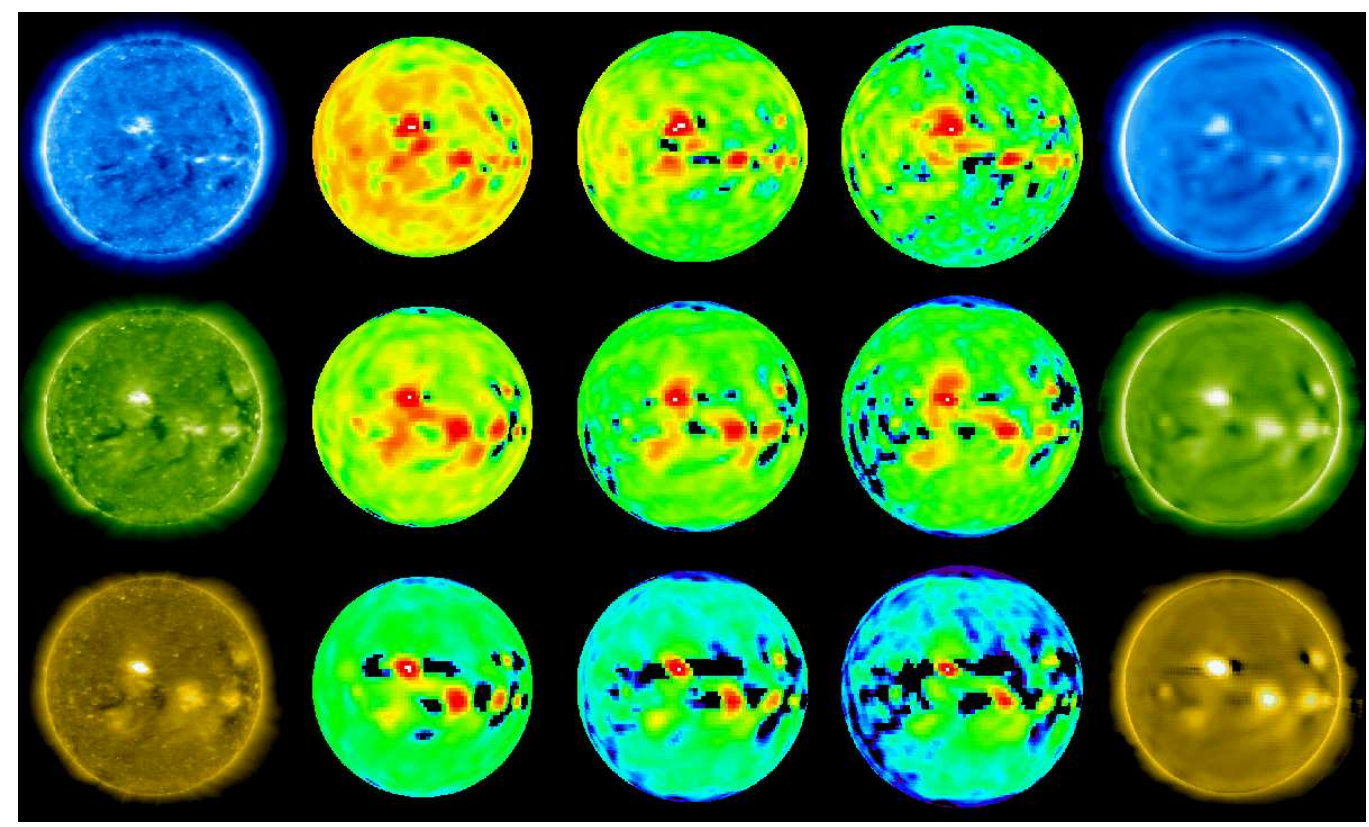

Figure 1: A summary of the EUV tomography. First column shows (from top to bottom) EUVI-A images in the 171, 195 and $284 \AA$ bands taken near 08:00 UT on 28 April 2008. For each band, the second through fourth columns show, in a color scale in the electronic version of this figure in the on-line version, projected spherical cuts of the tomographic FBE at $1.035,1.085$, and $1.135 R_{\odot}$, respectively. The last column shows the respective synthetic images calculated by integrating the tomographic models along the line-of-sight. The black streaks seen in the reconstructions near some of the active regions are artifacts caused by the Sun's temporal variability. From Vásquez et al. (2009).

To evaluate the accuracy of the tomographic model, the synthetic images can be quantitatively compared to the corresponding data images. An example of this, based on data from the AIA/SDO instrument, is shown in Figure 2 from a tomographic reconstruction of the solar corona in the bands of 171 , 193, 211, and $335 \AA$ (from top to bottom). 

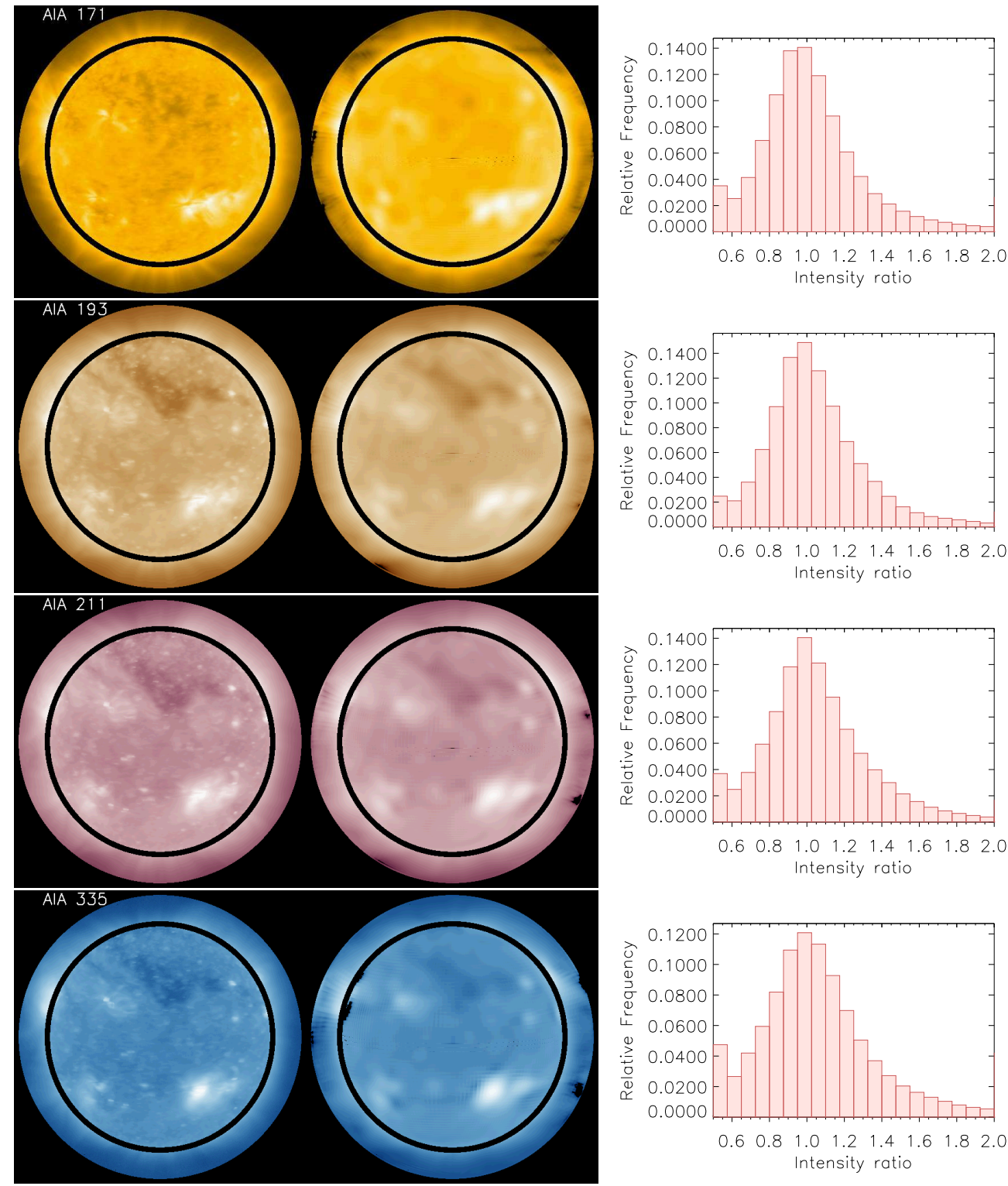

Figure 2: Comparison of synthetic data derived from the tomographic model against observed data in the bands of $171,193,211$, and $335 \AA$ (from top to bottom) of the AIA/SDO telescope. Left panels: data images and corresponding synthetic images computed by LOSintegration of the tomographic model. Right panel: frequency histogram of the synthetic to observed intensity ratio for every corresponding pair of pixels in the two images. 
The black rings in the images shown in Figure 2 correspond to pixels with projected radius in the range 0.98 to $1.025 R_{\odot}$. This near-limb data is not actually used for the tomographic inversion, as the emission along their corresponding line-of-sights can be affected by optically thick emission (Frazin et al., 2009). For each pair of images, the relative difference between the synthetic and observed values is below $0.1,0.2$ and 0.3 for about 35, 60 and $75 \%$ of the pixels, respectively. The same level of agreement holds for off-limb or on-disk pixels considered separately. The tomographic model provides then a quite detailed and reliable description of the average state of the global corona during the reconstructed period.

EUV tomography can currently be applied from only one or two (in the STEREO era) point-of-view. With such limited simultaneous information the temporal resolution of the technique is of the order of half solar rotation (or about two weeks). Of course, this is the most important limitation of the technique, which is then suitable for studying structures that are stable during their observed transit.

Thus far, all published work on DEMT has only used static SRT. However, time-dependent SRT based on Kalman filtering techniques has been applied to white light (WL) images of the corona (Butala et al., 2010). The type of detail and dynamics captured by EUV images is quite different to those seen in WL images. While for WL data time-dependent SRT has been shown to improve the reconstructions to some extent, it not clear what benefits would be obtained with EUV images.

\section{The 3D Distribution of the DEM}

Once the tomographic step is completed, the FBE of all EUV bands is known at each tomographic computational cell. Within each tomographic voxel the plasma is expected to be multi-thermal. The LDEM is a measure of the thermal distribution within the voxel.

As each FBE represents emission at a different temperature, they provide constraints to the determination of the LDEM. Using the passband function of each band of the EUV telescopes, and the atomic database CHIANTI version 7.1 to model of coronal emissivity, Figure 3 shows the temperature response function (TRF) of all EUVI and 4 AIA coronal bands. The temperature range modeled by the LDEM is determined by the sensitivity temperature range of the filters that are used. This can be estimated from the FWHM of the TRFSs around their respective main peaks. For EUVI and 


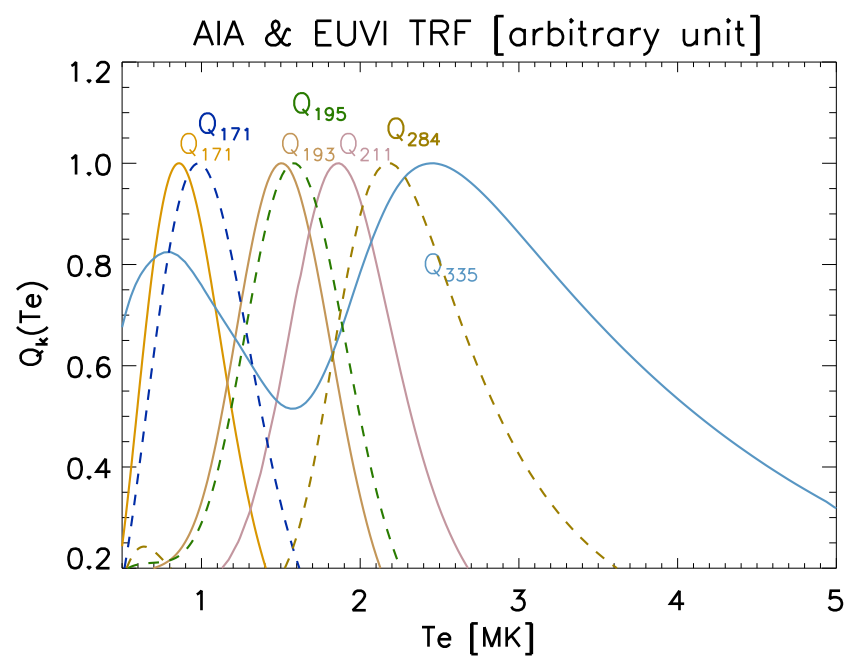

Figure 3: Temperature response function of all EUVI coronal bands (dashed, 171, 195, $284 \AA$ ), and 4 AIA coronal bands (solid, 171, 193, 211, $335 \AA$ ). From Nuevo et al. (2015).

the 3 lower temperature bands of AIA, the temperature range is from about 0.5 and up to about 3.0 MK. In the case of AIA, this range is expanded up to $\sim 4 \mathrm{MK}$ when adding the $335 \AA$ band (see Nuevo et al. (2015)).

Due to the limited number of available data points (FBE values), and also to the narrow band nature of the TRF of each band, the determination of the LDEM distribution is implemented by modeling it with a family of functions depending on a vector of a small number of parameters (typically 3 to 5 ). When DEMT is based on 3 bands, such as provided by the EUVI telescope, the LDEM is modeled by a single Gaussian function. With the AIA telescope, more coronal bands can be used (up to 6), and the LDEM can be modeled with combinations of Gaussian functions. The reader is referred to Nuevo et al. (2015) for a detailed study on parametric models of the LDEM using both telescopes.

In each tomographic cell independently, the problem consists in finding the values of the parameter vector to best predict with the LDEM the tomographic values of all FBEs in that cell. To do so an objective function is defined that measures the quadratic differences between tomographically determined FBEs and the those synthesized from the modeled LDEM.

Once the LDEM is determined, the average electron density in the to- 

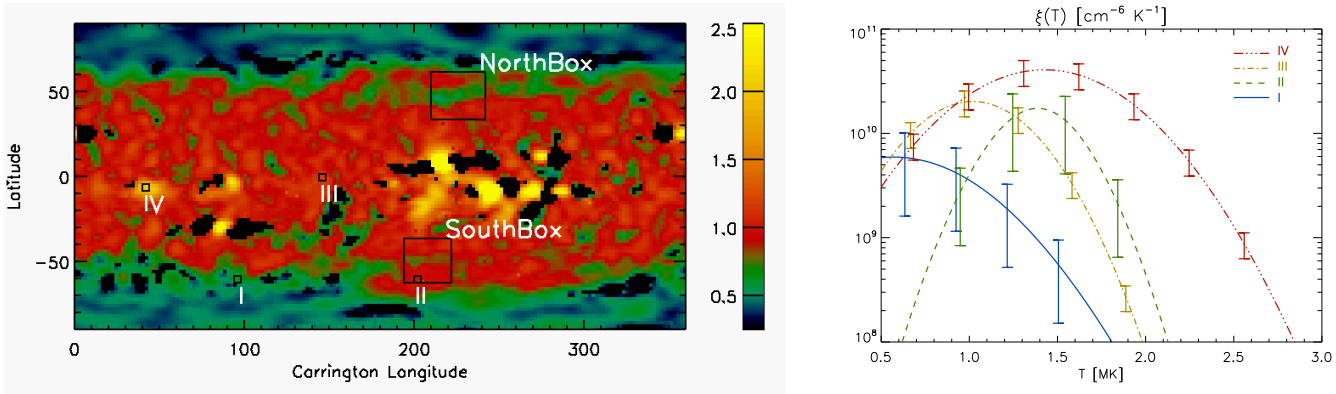

Figure 4: Left: Latitude-Longitude map of the tomographic electron density $N_{e}$ in units of $10^{8} \mathrm{~cm}^{-3}$, at a height $1.075 \mathrm{R}_{\odot}$, for the period CR-2069. Right: LDEM at the tomographic voxels I, II, III and IV indicated in the left panel. Reproduced from Frazin et al. (2009).

mographic cell can be computed from its zeroth moment, specifically as the square root of the integral of the LDEM over temperature. As an example, the left panel in Figure 4 shows a latitude-longitude map of a spherical shell of the electron density obtained at one sample height of the tomographic grid, for Carrington rotation (CR-)2069, a period of very low magnetic activity. At 4 tomographic voxels indicated as I, II, III, and IV in the left panel, the right panel shows the normal LDEM model that is found. The error bars represent the uncertainty due to the regularization level of the tomographic reconstructions. The first moment of the LDEM, divided by its zeroth moment, allows the computation of the mean electron temperature predicted by DEMT. Examples of spherical shells of the electron density and mean temperature at two different heights of another tomographic reconstruction are reproduced in Figure 5. Contour levels of the magnetic field strength $B$ of a potential field source surface (PFSS) model are over-plotted, along with the magnetically open/closed boundary (see caption in Figure 5).

\section{Results}

Applications of DEMT have included the observational study of coronal structures, the use of tomographic results to validate coronal models, and its combination with coronal extrapolations of the photospheric magnetic field. Following we summarize all peer-reviewed published work on DEMT.

Vásquez et al. (2009) produced the first observational 3D analysis of stable coronal prominence cavities, measuring the density and temperature contrast between the plasmas in the cavity and in the surrounding helmet streamer. 

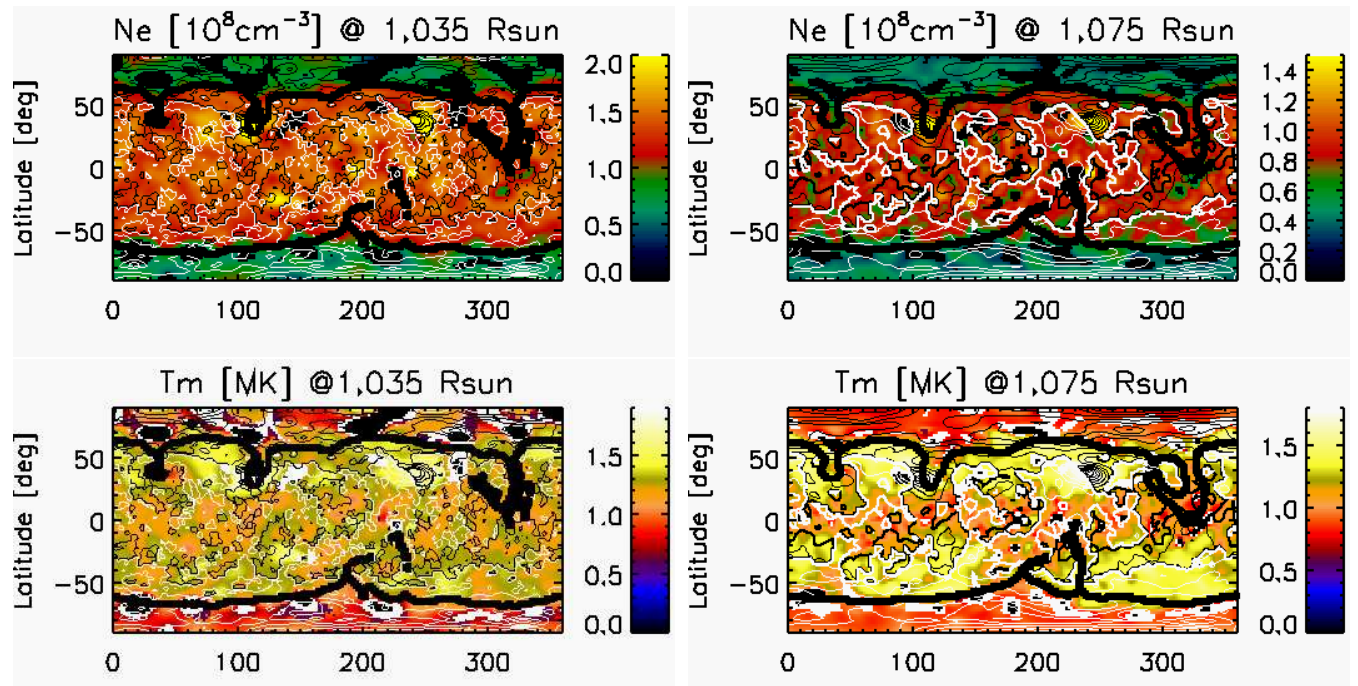

Figure 5: An example of the 3D reconstruction of the thermodynamical state of the solar minimum corona, during the period CR-2077. Latitude-longitude maps of the electron density (top) and mean electron temperature (bottom) at heights 1.035 (left) and 1.075 $R_{\odot}$ (right), as derived with the DEMT technique. Solid-thin curves are magnetic field strength $B$ contour levels of a PFSS model of the coronal magnetic field, with white (black) representing outward (inward) oriented magnetic field. The solid-thick black curves indicate the location of the magnetically open/closed boundary.

As it is characteristic of tomography, their study did not require any ad-hoc modeling, as needed in the forward modeling approach.

Being suited to study coronal structures that are stable over half a solar rotational time, tomography works best at solar minimum. Vásquez et al. (2010, 2011) analyzed the global thermodynamics of the solar corona during the minimum of activity between solar cycles 23 and 24, and discussed their results in relation to the fast and slow components of the solar wind. These works also include comparisons to other observational non-tomographic studies of the same periods, providing cross validation results for the technique. As an example of a 3D reconstruction of the solar minimum, Figure 5 displays latitude-longitude maps of the electron density and mean temperature, derived with the DEMT technique for the period CR-2077. At both heights, the location of the open/closed boundary of the PFSS model is characterized by a very high transverse gradient in both the electron density and the mean temperature maps derived from the DEMT analysis. 

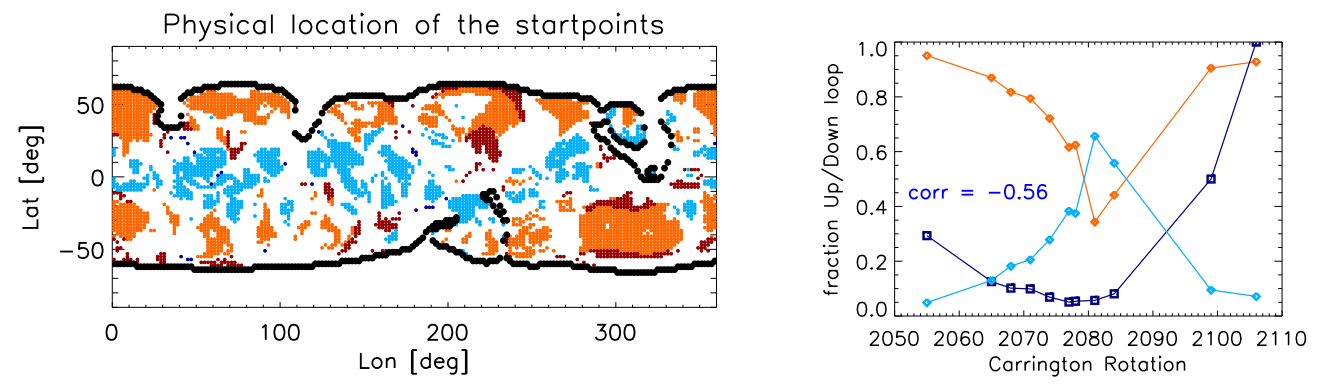

Figure 6: Note: all indication of colors refers to the online version of this figure. Left: Location of loops with negative (down) and positive (up) temperature gradient with height, in blue and red, respectively, for CR-2077. Right: Evolution of the fraction of down (up) loops indicated as light-blue (red) diamonds, and the sunspot monthly number divided by 32.1 (dark-blue squares). The linear correlation coefficient between the fraction of down loops and the sunspot number is indicated. Adapted from Nuevo et al. (2013a).

DEMT can be combined with the global PFSS magnetic models of the solar corona, an approach dubbed the Michigan Loop Diagnostic Technique (MLDT). DEMT results are traced along the field lines of the magnetic model, allowing study of the thermodynamical properties of magnetic flux tubes in the quiet Sun in a statistical fashion. Huang et al. (2012) applied the MLDT to study one rotation during the last solar minimum. They found the ubiquitous presence of magnetic loops with downward gradients of temperature dubbed down loops. Down loops were found to be dominant in the latitude range $\pm 30^{\circ}$ (left panel in Fig. 6). Nuevo et al. (2013a) extended the study to a sequence of rotations that included the solar minimum. Their study revealed a clear anti-correlation between the global coronal activity level and the number of down loops present in the corona (right panel in Fig. 6). They found that down and up loops are characterized $\beta \approx 1$ and $\beta<1$, respectively, and proposed an interpretation of their results in terms of Alfvén wave damping.

DEMT results have been also used to constrain and validate MHD models. DEMT reconstructions of the electron density and temperature have been used as a constraint of a two-temperature 3D MHD solar wind model of the Space Weather Modeling Framework (SWMF) code suite (van der Holst et al., 2010). Improvements on the performance of the models include a more accurate prediction of the occurrence and density of co-rotating interaction regions in the heliosphere. 


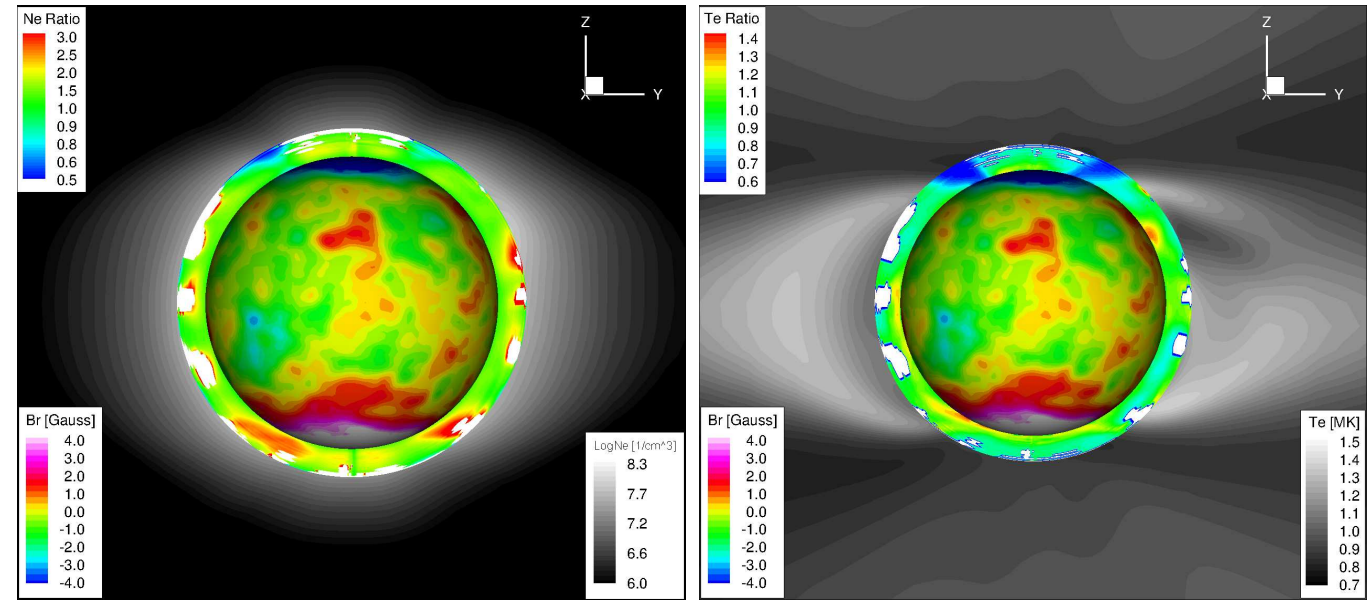

Figure 7: Note: all indication of colors refers to the online version of this figure. DEMT validation of the electron density (left) and temperature (right) of a two-temperature MHD model of the solar corona, for the period CR-2077. The color rings spanning the height range 1.0 to $1.25 R_{\odot}$ show, in a sample meridional cut of the corona, the MHD to DEMT ratio of the respective quantity. The outer greyscale maps $\left(r>1.25 R_{\odot}\right)$ display the corresponding MHD results. Reproduced from Jin et al. (2012).

Global two-temperature models of the corona and the heliosphere have been validated in the inner part of the corona with DEMT reconstructions of the electronic plasma parameters (Jin et al., 2012), with most of the model outputs fitting the observations very well, as seen in Figure 7 . The 3D products of DEMT have also been used as a validation tool for a study on coronal heating by surface Alfvén wave damping, implemented within the MHD model of the solar wind in the SWMF (Evans et al., 2012). DEMT results have been recently used as a validation tool in a study of the charge state composition of the slow solar wind derived from an ionization evolution code coupled to the wave-driven MHD solar wind model of the SWMF (Oran et al., 2014). DEMT allowed detailed validation of the latitudinal transition of the electronic plasma parameters in the open field lines surrounding the coronal equatorial streamer belt (Fig. 8).

All DEMT studies reviewed so far were based on EUVI data, having 3 EUV bands with a sensitivity range $\sim 0.60-2.70 \mathrm{MK}$. More recently, Nuevo et al. $(2013 \mathrm{~b}, 2015)$ extended the DEMT technique to use the 4 cooler AIA bands (aimed at studying the quiet Sun), sensitive to the range $\sim 0.55-3.75$ MK. Their study corresponds to CR-2099, a rotation of the rising phase of 


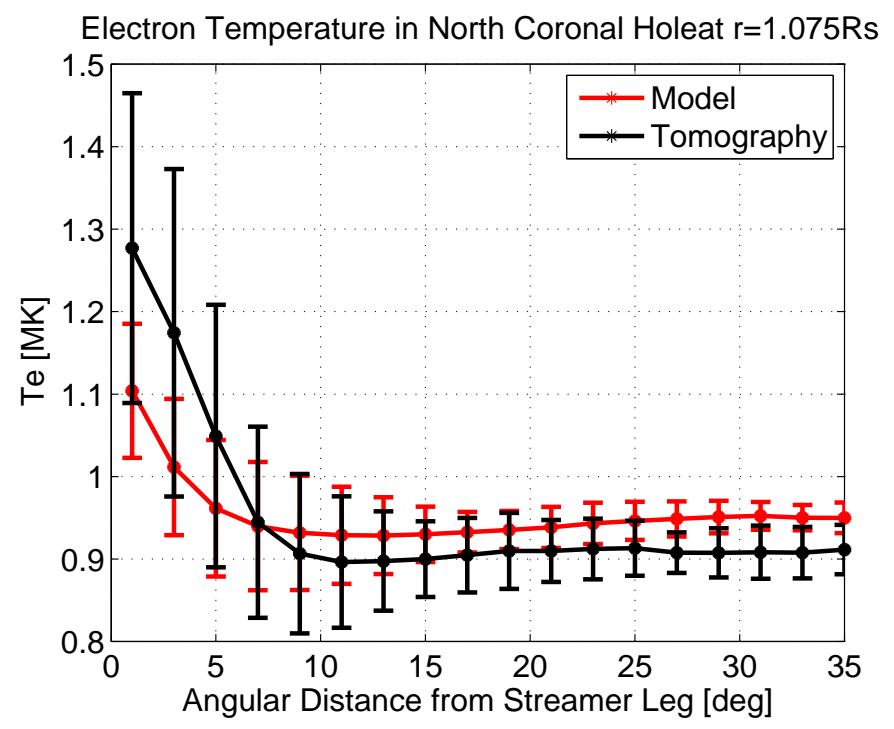

Figure 8: Note: all indication of colors refers to the online version of this figure. DEMT validation of a 3D wave-driven MHD model of the solar wind. Electron temperature versus angular distance from the magnetically open/closed boundary (averaged over all longitudes) for the period CR-2063, derived from the MHD model (red) and from the DEMT reconstruction (black). Reproduced from Oran et al. (2014).

the current solar cycle 24. While in previous studies the LDEM was always modeled by a single Gaussian distribution, the extra AIA filter, and the increased sensitivity range, allowed exploration of new parametric LDEM models. Using 4 bands, the model that consistently achieves the best predictedto-reconstructed FBEs is a bimodal distribution, being a superposition of two Gaussian distributions with distinct warm and hot components. The mean centroids of the two components in the quiet diffuse corona are found to be $\log _{10}\left\langle T_{0,1}\right\rangle=6.15$ and $\log _{10}\left\langle T_{0,2}\right\rangle=6.42$, values that are very consistent with independent determinations of the characteristic temperatures of the solar corona, as discussed in the same study.

The square of the electron densities of the two components are $N_{e, 1}^{2}$ and $N_{e, 2}^{2}$, respectively, so that the total square electron density of the LDEM is $N_{e}^{2}=N_{e, 1}^{2}+N_{e, 1}^{2}$. A measure of the bi-modality is then the ratio $\left(N_{e, 2} / N_{e}\right)^{2}$. The study of this ratio throughout the diffuse quiet corona reveals that the bimodality of the LDEM is ubiquitous, and that it is stronger for denser regions, as shown by the left panel of Figure 9 . 
Nuevo et al. (2015) also validated the LDEM determination technique by applying it to standard 2D DEM studies. Examples of the bimodal DEMs are shown in the right panel of Figure 9. The DEMT study shows that the LDEM of the quiet corona is bimodal at the spatial resolution of the tomographic grid, which is $0.01 R_{\odot} \times 2^{\circ} \times 2^{\circ}$, or about $\left(7 \times 10^{3} \mathrm{~km}\right) \times\left[\left(2.44 \times 10^{4} \mathrm{~km}\right)^{2}\right]$ for a representative voxel at a height of $0.1 R_{\odot}$ above the photosphere at the equator. The authors argue that the nanoflare heating scenario is less likely to explain these results, and that alternative mechanisms, such as wave dissipation, appear better supported by them.
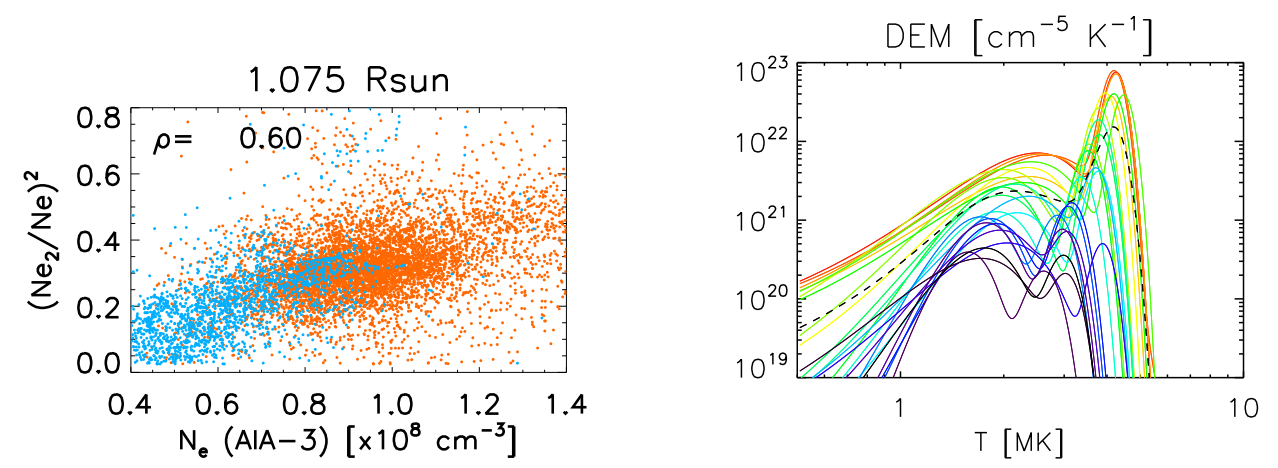

Figure 9: Note: all indication of colors refers to the online version of this figure. Left: Scatter plot of the measure of the bi-modality $\left(N_{e, 2} / N_{e}\right)^{2}$ (see text) versus $N_{e}$ derived with AIA, at $1.075 R_{\odot}$. Right: Bi-modal DEM curves obtained for selected regions within and around an active region. Red/yellow curves correspond to hotter and denser regions, blue/dark curves correspond to colder and less dense regions. Reproduced from Nuevo et al. (2015).

\section{Concluding Remarks and Future Prospects}

DEMT provides a quantitative description of the average state of the solar corona over a full solar rotation with the following main characteristics,

- The products are 3D maps of: a) the filter band emissivity in each EUV band, b) the LDEM, and c) its moments, such as $\left\langle N_{e}^{2}\right\rangle$ and $\left\langle T_{e}\right\rangle$.

- The temporal resolution of the technique is currently limited to the transit time of coronal features, i.e. $\sim 14$ days. DEMT reconstructions 
are then reliable descriptions of slowly evolving coronal structures, such as the diffuse quiet corona, and coronal holes. DEMT is not suited to study regions characterized by fast dynamics.

- The spatial resolution depends on the computational grid size, which in turn is constrained by the cadence of the image series. Typically, one image every 6 hrs is used, a time over which the Sun rotates about $3.3^{\circ}$. The adopted tomographic cell size is then $2^{\circ}$ in both angular directions and $10^{-2} R_{\odot}$ in the radial direction.

- The image processing, tomographic inversion, and DEM determination, are fully automated tasks, with little user interaction.

- Its implementation does not require any ad-hoc modeling.

- Finite FOV and finite computational grid effects, issues of relevance for white light tomography, are generally not important in EUV tomography due to the typical size of the FOV in full-sun images of the EUV telescopes and the $N_{e}^{2}$ scaling of the EUV emissivity.

The current implementation of the DEMT and MLDT techniques involves a suite of codes written in the $\mathrm{C}$ and IDL programming languages, which can be efficiently ran in nowadays desktop multi-core computers. Future immediate planned applications of DEMT and the MLDT involve comparative studies of the last two solar minima (using the EIT and EUVI instruments), as well as the study of the coronal radiative losses predicted by the MLDT in the quiet Sun.

Up to this point DEMT has been based in static SRT, but future work may include extending the technique to include time-dependence (Butala et al., 2010). Tomographic inversion of time series of full-sun images is the only available observational technique that can provide global constraint and validation to large scale MHD modeling of the corona and the solar wind. As such, DEMT is a highly valuable tool to help in the continued development of global coronal models. DEMT could provide highly valuable 3D maps of the detailed temperature distribution of the coronal plasma if full-sun spectral images (of the type provided by the EIS instrument) become available. It would be highly desirable that such an instrument will be operational during the a solar minimum period, when single point-of-view tomography is most fruitful. 
The author thanks CONICET grant PIP IU Nro 11420100100151 that has funded this research. The author also thanks the collaboration and useful comments by Richard A. Frazin and Federico A. Nuevo.

Altschuler, M. D., Perry, R. M., Apr. 1972. On Determining the Electron Density Distribution of the Solar Corona from K-Coronameter Data. Solar Physics, 23, 410-428.

Aschwanden, M. J., Oct. 2011. Solar Stereoscopy and Tomography. Living Reviews in Solar Physics 8, 5.

Butala, M. D., Hewett, R. J., Frazin, R. A., Kamalabadi, F., Apr. 2010. Dynamic Three-Dimensional Tomography of the Solar Corona. Solar Physics, 262, 495-509.

Evans, R. M., Opher, M., Oran, R., van der Holst, B., Sokolov, I. V., Frazin, R., Gombosi, T. I., Vásquez, A., Sep. 2012. Coronal Heating by Surface Alfvén Wave Damping: Implementation in a Global Magnetohydrodynamics Model of the Solar Wind. The Astrophysical Journal, 756, 155.

Frazin, R. A., Feb. 2000. Tomography of the Solar Corona. I. A Robust, Regularized, Positive Estimation Method. The Astrophysical Journal, 530, 1026-1035.

Frazin, R. A., Janzen, P., May 2002. Tomography of the Solar Corona. II. Robust, Regularized, Positive Estimation of the Three-dimensional Electron Density Distribution from LASCO-C2 Polarized White-Light Images. The Astrophysical Journal, 570, 408-422.

Frazin, R. A., Kamalabadi, F., Weber, M. A., Aug. 2005. On the Combination of Differential Emission Measure Analysis and Rotational Tomography for Three-dimensional Solar EUV Imaging. The Astrophysical Journal, 628, 1070-1080.

Frazin, R. A., Vásquez, A. M., Kamalabadi, F., Aug. 2009. Quantitative, Three-dimensional Analysis of the Global Corona with Multispacecraft Differential Emission Measure Tomography. The Astrophysical Journal, 701, 547-560. 
Huang, Z., Frazin, R. A., Landi, E., Manchester, W. B., Vásquez, A. M., Gombosi, T. I., Aug. 2012. Newly Discovered Global Temperature Structures in the Quiet Sun at Solar Minimum. The Astrophysical Journal, 755, 86.

Inhester, B., Dec. 2006. Stereoscopy basics for the STEREO mission. ArXiv Astrophysics e-prints.

Jin, M., Manchester, W. B., van der Holst, B., Gruesbeck, J. R., Frazin, R. A., Landi, E., Vasquez, A. M., Lamy, P. L., Llebaria, A., Fedorov, A., Toth, G., Gombosi, T. I., Jan. 2012. A Global Two-temperature Corona and Inner Heliosphere Model: A Comprehensive Validation Study. The Astrophysical Journal, 745, 6.

Leblanc, Y., Leroy, J. L., Poulain, P., May 1970. The Characteristic Sizes and the Electron Density of Coronal Enhancements Observed in White Light. Astronomy and Astrophysics, 5, 391.

Minnaert, M., 1930. On the continuous spectrum of the corona and its polarisation. Zeitschrift für Astrophysik, 1, 209.

Nuevo, F. A., Huang, Z., Frazin, R., Manchester, iv, W. B., Jin, M., Vásquez, A. M., Aug. 2013a. Evolution of the Global Temperature Structure of the Solar Corona during the Minimum between Solar Cycles 23 and 24. The Astrophysical Journal, 773, 9.

Nuevo, F. A., Vásquez, A. M., Frazin, R. A., Landi, E., 2013b. Multi-modal DEM in the solar corona. Boletin de la Asociacion Argentina de Astronomia La Plata Argentina 56, 395-398.

Nuevo, F. A., Vásquez, A. M., Landi, E., Frazin, R., 2015. Multimodal Differential Emission Measure in the Solar Corona. The Astrophysical Journal, submitted.

Oran, R., Landi, E., van der Holst, B., Lepri, S. T., Vásquez, A. M., Nuevo, F. A., Frazin, R., Manchester, IV, W. B., Sokolov, I. V., Gombosi, T. I., Dec. 2014. A Steady-State Picture of Solar Wind Acceleration and Charge State Composition Derived from a Global Wave-Driven MHD Model. ArXiv e-prints. 
van de Hulst, H. C., Feb. 1950. The electron density of the solar corona. Bulletin of the Astronomical Institutes of the Netherlands, 11, 135.

van der Holst, B., Manchester, IV, W. B., Frazin, R. A., Vásquez, A. M., Tóth, G., Gombosi, T. I., Dec. 2010. A Data-driven, Two-temperature Solar Wind Model with Alfvén Waves. The Astrophysical Journal, 725, 1373-1383.

Vásquez, A. M., Frazin, R. A., Kamalabadi, F., May 2009. 3D Temperatures and Densities of the Solar Corona via Multi-Spacecraft EUV Tomography: Analysis of Prominence Cavities. Solar Physics, 256, 73-85.

Vásquez, A. M., Frazin, R. A., Manchester, IV, W. B., Jun. 2010. The Solar Minimum Corona from Differential Emission Measure Tomography. The Astrophysical Journal, 715, 1352-1365.

Vásquez, A. M., Huang, Z., Manchester, W. B., Frazin, R. A., Dec. 2011. The WHI Corona from Differential Emission Measure Tomography. Solar Physics, 274, 259-284. 